\title{
Multiple phase-shift all-fibre DFB lasers
}

\author{
N.Y.Voo and M. Ibsen \\ Optoelectronics Research Centre, University of Southampton, Southampton SO17 1BJ, United Kingdom
}

\begin{abstract}
A double $(2 \times \pi / 2)$ phase-shifted Er/Yb fibre DFB laser shows a 50\% reduction of the shift in lasing wavelength and a $10 \%$ reduction of the linewidth, compared to standard single phase-shifted fibre DFB designs.
\end{abstract}

\section{Introduction}

Short cavity single frequency fibre lasers have been a topic of continued interest since the early work of Ball et al on Er-doped distributed Bragg reflector (DBR) fibre lasers [1]. Their size, simple design, ease of fabrication, compatibility to transmission fibres and optical emission characteristics make them attractive for a whole host of applications, particularly in the communications and sensing areas. A number of distributed feedback (DFB) laser cavity designs have been proposed and demonstrated in the literature in both semiconductor [2, 3] and fibre hosts. These include uniform, non phase-shifted [2], multiple phase-shifted [3], symmetric single $\pi$ phase-shifted [4-6], asymmetric single $\pi$ phase-shifted [7-9] and step-apodized [10] designs, each offering different performance and distinctive operational characteristics.

In this paper, we demonstrate a $2 \times \pi / 2$ phase-shifted fibre DFB laser for the first time and analyse its performance against the performance benchmark of a single asymmetric $\pi$-phase shifted DFB fibre laser [7-9]. Through directly comparing the key operational parameters of the lasers such as output power, shift of wavelength against pump power, linewidth and relative intensity noise (RIN), we find that for a similar efficiency, the multiple phase-shifted laser exhibits a slight increase in amplitude noise at the lowest output powers. However, more importantly it exhibits a reduction in the relative lasing wavelength shift against pump of about $50 \%$ and a $\sim 10 \%$ reduction in the linewidth, suggesting a more uniform intensity distribution within the laser cavity.

\section{Experimental details and results}

The fibre DFB lasers considered in this study are constructed within a $125 \mu \mathrm{m}$ diameter fibre containing a highly doped Er-Yb phosphosilicate core with an annular photosensitive boron ring to the core [11]. The $50 \mathrm{~mm}$ long laser cavities were UV-written with a Bragg grating wavelength of $1552 \mathrm{~nm}$ and the small signal absorption in the fibre is $1200 \mathrm{~dB} / \mathrm{m}$, and $45 \mathrm{~dB} / \mathrm{m}$, at $980 \mathrm{~nm}$ and $1530 \mathrm{~nm}$ respectively. Both lasers have a coupling coefficient $\sim 200 \mathrm{~m}^{-1}$ with the position of the discrete phase shifts for the two $\pi / 2$ phase-shifted laser being $5 \mathrm{~mm}$ apart at positions 19.5 $\mathrm{mm}$ and $24.5 \mathrm{~mm}$ respectively and at $22 \mathrm{~mm}$ for the asymmetric $\pi$ phase-shifted DFB laser. The lasers were pumped with a 980 -nm grating-stabilized laser diode and positioned on a temperature-controlled heat sink during testing with the linewidth measurements being performed using a self-heterodyne technique with a $60 \mathrm{~km}$ fibre delay line.

The performance of the lasers was investigated in two pump configurations. In the backward pumpconfiguration, the pump is counter-propagating to the laser output, i.e. the pump is launched at the end closest to the phase-shift. In the forward pump-configuration the pump and signal are co-propagating, meaning that the pump is coupled in at the end furthest away from the grating phase-shift. A 980-nm optical isolator is employed at the output of the pump laser, and 1550-nm isolators at the fibre laser outputs, to prevent feedback effects. The lasing wavelength shift with output power was measured with a high-precision wave-meter, and the linewidth and RIN with a Lightwave analyser. Furthermore, the polarisation state of the lasers was investigated with a polarisation analyser with which both lasers were confirmed to be operating in a single polarization.

The output characteristics of the DFB lasers for backward and forward pumping configurations are shown in Fig.1. As indicated, the lasing threshold for the double $\pi / 2$ phase-shifted DFB under forward pumping, is slightly higher than the asymmetric $\pi$ phase-shifted design. Because of the very high pump absorption and the phase-shifts for the double phase-shifted design are $5 \mathrm{~mm}$ apart, the round trip feedback is longer resulting in a slightly increased lasing threshold. However, once lasing, the slope efficiencies for both lasers are similar. Backward pumping is found to be the marginally more power efficient in both DFB cases. In this configuration, the slope efficiencies of the double and single phase-shifted designs are $20 \%$ and $21.2 \%$ respectively and $19.1 \%$ and $18.1 \%$ respectively in the forward configuration. 


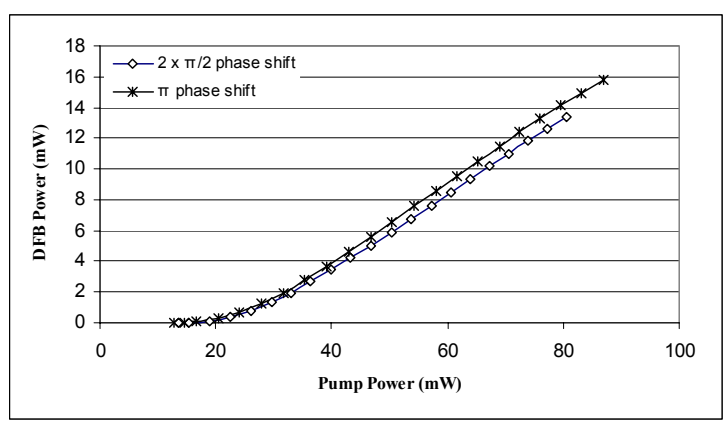

(a)

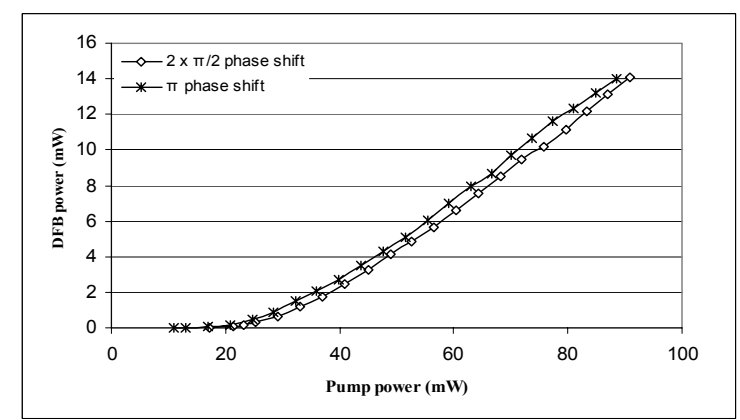

(b)

Fig. 1. DFB lasers output power versus pump power for (a) backward- and (b) forward- pumping configurations.

Internal heating of fibre lasers due to the dissipated pump power has been discussed in detail in [12]. For our lasers, the pump to signal conversion is about $\sim 20 \%$ suggesting that some of the pump is turned into heat, which in turn would raises the fibre temperature, resulting in a refractive index increase of the entire fibre and more specifically the fibre core because of the positive thermo-optic coefficient for silica and phosphosilica. This increase therefore would cause a shift in the Bragg wavelength of the DFB grating. However, it is unclear if all the measured wavelength shift is a result of the pump caused temperature increase only, or if there is also an intra-cavity effect arising from the high signal intensity around the phase-shift it self. Previously no experimental results supporting or separating these effects have been demonstrated. For that reason, we measured the lasing wavelength as a function of pump power to investigate this point further. Fig. 2(a) and (b) shows for the backward and forward pump configurations respectively, that the wavelength increases by only $\sim 17 \mathrm{pm}$ and $\sim 18 \mathrm{pm}$ for the double $\pi / 2$ phaseshifted DFB laser as opposed to $\sim 35 \mathrm{pm}$ and $\sim 40 \mathrm{pm}$ for single $\pi$ phase-shifted DFB over an increase of pump power of $\sim 80 \mathrm{~mW}$. This very strongly suggests that the intra-cavity intensity contributes to $\sim 50 \%$ of the wavelength shift fo a similar efficiency of the lasers (Fig.1). This design might therefore also open up the possibility of power-scaling of fibre DFB's because the intra-cavity powers are kept at a minimum and hence the risk of cavity dephasing is significantly reduced.

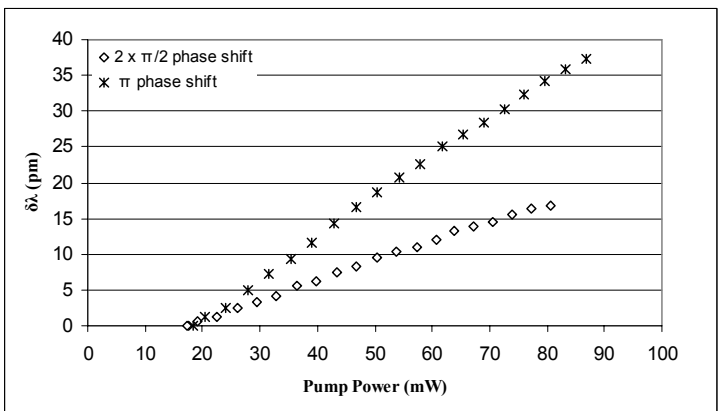

(a)

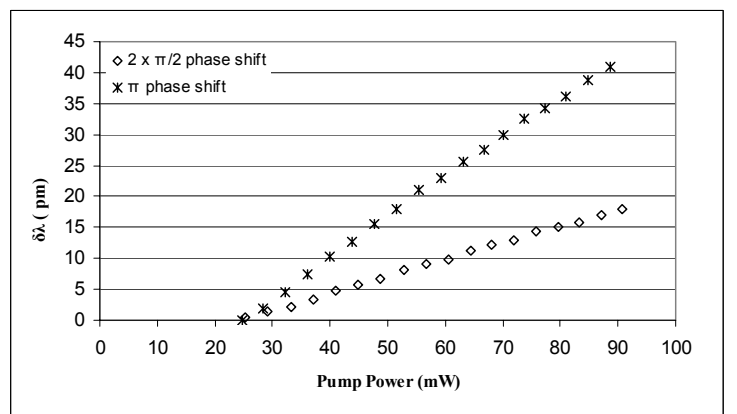

(b)

Fig. 2. Lasing wavelength shift $(\delta \lambda)$ against pump power for (a) backward- (b) forward- pumping configurations.

Because of our previous work of establishing a link between the sensitivity of the cavity to variations of the pump-power and the linewidth of the lasers [9], we also measure the linewidth of these devices. Those results are summarised in Fig. 3 where the 3-dB laser linewidth behaviour as a function of pump power for the two pumping schemes is plotted. For the backward pumping-configuration, the data demonstrate that the linewidth obtained from the double phase-shifted laser is $\sim 10 \%$ lower than the single phase-shifted laser. Its linewidth exhibits an increase from $21-37 \mathrm{kHz}$ over the pump power range. For the forward pumping configuration, the difference is not as marked, but the double phase-shifted laser still shows a lower linewidth for an output power up to $\sim 6 \mathrm{~mW}$, above which the two laser designs exhibits a similar linewidth of $\sim 60 \mathrm{kHz}$. It is not clear why the difference in linewidth is only $\sim 10 \%$ when the differences of the lasing wavelength with pump power of the two designs shows such a dramatic reduction. We hope to be in a position to shed more light on that at the time of the conference. 


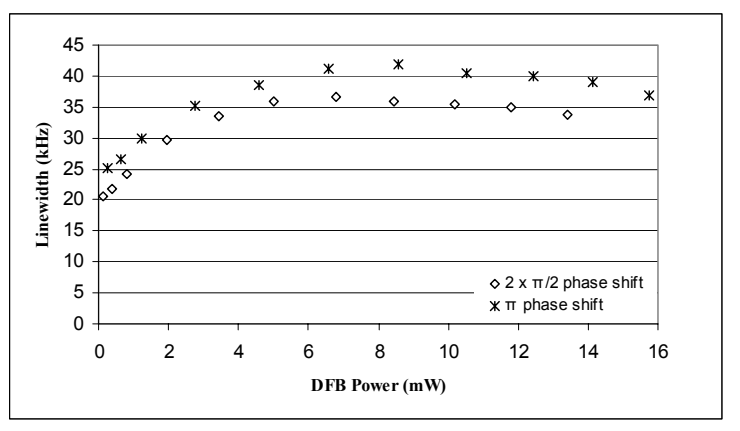

(a)

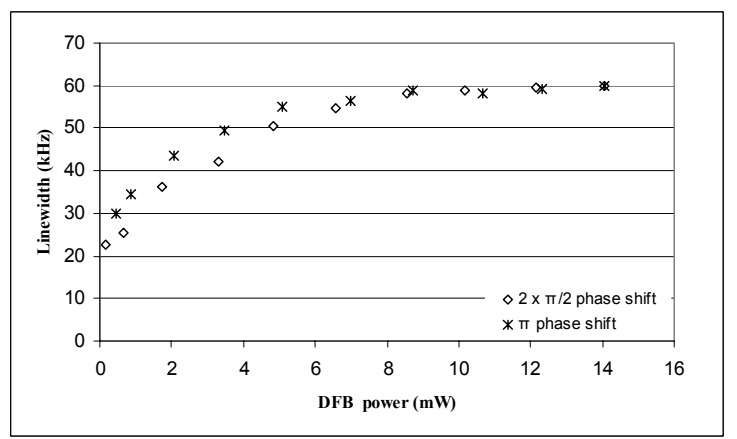

(b)

Fig. 3. 3-dB laser linewidth versus pump power for (a) backward- and (b) forward- pumping configurations.

The relative intensity noise (RIN) and the relaxation oscillation (RO) frequency of the lasers as a function of DFB power was also investigated (Fig. 4). There is a quite different RIN performance of the laser designs under the considered pump conditions with the double phase-shifted laser exhibiting a lower RIN at the RO-frequency peak for output powers above $\sim 5 \mathrm{~mW}$ in the backward pumping scheme. In the forward pumping configuration, the RIN performance of the standard single phase-shifted laser is lower for all output power levels. The reason for this amplitude noise behaviour is not yet fully understood, but both designs approach a RIN level below $-125 \mathrm{~dB} / \mathrm{Hz}$ at the RO frequency for powers above $\sim 13 \mathrm{~mW}(11 \mathrm{dBm})$ in the backward pump configuration.

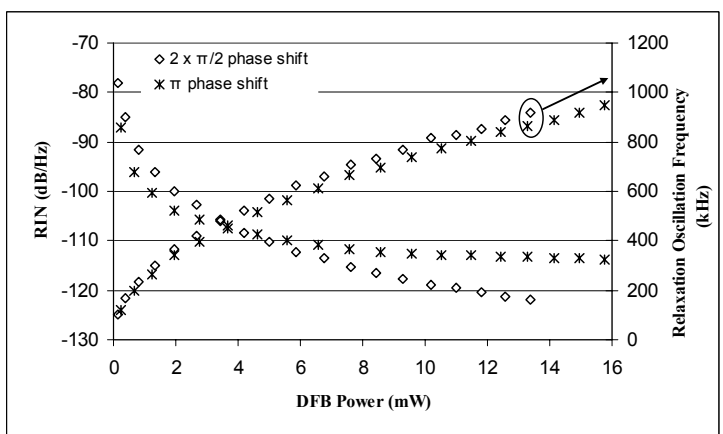

(a)

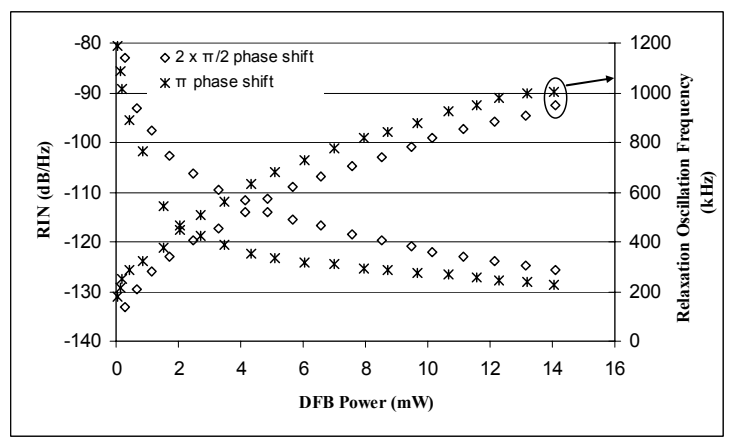

(b)

Fig. 4. DFB lasers' intensity noise versus DFB power for (a) backward- and (b) forward- pumping configurations.

\section{Conclusions}

We have experimentally demonstrated a double $\pi / 2$ phase-shifted all-fibre DFB laser for the first time, analysed, and compared its performance and key operational parameters against the performance of a single $\pi$ phase-shifted DFB laser. Remarkably, we have found that the pump-induced lasing wavelength shift is reduced by $50 \%$ in the double phase-shifted laser with a resulting reduction in the linewidth of the laser as well. We believe that our results suggest that the application of multiple phase-shifting in DFB lasers can improve their performance and assist in the understanding and tailoring of key operational parameters of the lasers.

\section{References}

[1] G.A. Ball et al., "Standing-wave monomode erbium fiber laser," IEEE Photon. Technol. Lett. 3, 613-615 (1991).

[2] H. Kogelnik and C.V. Shank,"Coupled-wave theory of distributed feedback lasers," J. Appl. Phys. 43, 2327-2335 (1972).

[3] J. Karol et al., 'Distributed feedback semiconductor lasers', SPIE Press and IEE London, 1998.

[4] J.T. Kringlebotn et al., " $\mathrm{Er}^{3+} \mathrm{Yb}^{3+}$ codoped fiber distributed-feedback laser," Opt. Lett. 19, 2101-2103 (1994).

[5] W.H. Loh and R.I. Laming, "1.55 $\mu \mathrm{m}$ phase-shifted distributed feedback fiber laser,” Electron. Lett. 31, 1440-1442 (1995).

[6] M.Sejka et al., "Distributed feedback $\mathrm{Er}^{3+}$ doped fiber laser," Electron. Lett. 31, 1445-1446 (1995).

[7] V.C. Lauridsen, et al., "Design of DFB fiber lasers," Electron. Lett. 34, 2028-2030 (1998).

[8] M. Ibsen et al., "Robust high power $(>20 \mathrm{~mW})$ all fiber DFB lasers with unidirectional and truly single polarization outputs," in proceedings to CLEO'99, paper CWE4, (1999).

[9] N.Y. Voo et al., "Anomalous linewidth behaviour in short-cavity single-frequency fiber lasers," IEEE Photon. Technol. Lett. 17, 546-548 (2005).

[10] K. Yelen et al., “A new design approach for fiber DFB lasers with improved efficiency,” IEEE J. Quantum Electron. 40, 711-720 (2004).

[11] L. Dong et al., "Efficient single frequency laser with novel photosensitive Er/Yb optical fibers," Opt. Lett. 22, 694-696 (1997).

[12] M.K. Davis et al., "Thermal effects in doped fibers," J. Lightwave Technol. 16, 1013-1023 (1998). 